Original Paper http://ajol.info/index.php/ijbcs http://indexmedicus.afro.who.int

\title{
Caractéristique structurale des zones marécageuses de la forêt de la Tanoé-Ehy (Sud-Est de la Côte d'Ivoire)
}

\author{
Koffi MISSA ${ }^{1,2^{*}}$, Fofana SEGUENA ${ }^{3}$, Dramane SORO $^{2,3}$ et Adama BAKAYOKO ${ }^{2,4}$ \\ ${ }^{1}$ Université Nangui Abrogoua. Centre de Recherche en Ecologie (CRE), 02 BP 801 Abidjan 02, Côte d'Ivoire. \\ ${ }^{2}$ Centre Suisse de Recherches Scientifiques en Côte d'Ivoire, 01 BP 1303, Abidjan, Côte d'Ivoire. \\ ${ }^{3}$ Université Peleforo Gon Coulibaly, Institut de Gestion Agropastorale, BP 1328 Korhogo, Côte d'Ivoire \\ ${ }^{4}$ Université Nangui Abrogoua, UFR des Sciences de la Nature (SN) Côte d'Ivoire 02 BP 801 Abidjan 02. \\ *Auteur correspondant ; E-mail : botamissa@gmail.com
}

\section{REMERCIEMENTS}

La présente étude s'inscrit dans le cadre d'un processus de conservation, de protection et de préservation de la FMTE piloté par l'ONG Recherche et Action des Primates de Côte d'Ivoire.

\section{RESUME}

Cette étude vise à dégager les différents états de dégradation de la végétation au sein de la forêt marécageuse de la Tanoe-Ehy, à travers 4 relevés linéaires de 100 mètres de long. L'analyse de ces relevés a montré qu'au niveau des types biologiques, l'espèce Combretum racemosum P. Beauv. (Combretaceae) représente l'essentielle des espèces lianescentes. Dans cette forêt, parmi les espèces qui contribuent fortement à la formation de la végétation, il y a la présence des espèces vulnérables de l'UICN. Concernant les recouvrements, les strates basses sont faiblement occupées. Cependant, la végétation est dense dans les strates moyennes. Ces recouvrements ont montré que la dégradation de la structure des milieux est une conséquence de l'exploitation villageoise. Par ailleurs, cette forêt marécageuse présente des recouvrements particuliers qui n'ont jamais été observés en forêt dense humide de Côte d'Ivoire.

(C) 2020 International Formulae Group. All rights reserved.

Mots clés : Structure, végétation, forêt, marécageuse, types biologiques, recouvrements.

\section{Structural Characteristic of the marshy areas of the Tanoé-Ehy forest (Southeastern Côte d'Ivoire)}

\begin{abstract}
This study aims to identify the different state of vegetation degradation within the Tanoe-Ehy swamp forest, through 4 linear surveys 100 meters long. Analysis of these surveys showed that at the level of biological types, the species Combretum racemosum P. Beauv. (Combretaceae) is the essential of lianescent species. In this forest, among the species that contribute heavily to vegetation formation, there is the presence of vulnerable IUCN species. For overlays, the lower strata are poorly occupied. However, vegetation is dense
\end{abstract}


in the middle strata. These recoveries have shown that the degradation of the structure of the environments is a consequence of village exploitation. In addition, this swamp forest has special overlays that have never been seen in the dense rainforest of Côte d'Ivoire.

Keywords: Structure, vegetation, forest, swampy, biological types, recoveries.

\section{INTRODUCTION}

Dans les pays africains, la conservation et la protection de la biodiversité végétale s'avère plus difficile à appliquer en dehors des aires protégées où les forêts sont facilement affectées par diverses activités anthropiques (Gueulou et al., 2012). En Côte d'Ivoire, pour mieux diriger la politique de conservation et de protection, il devient de plus en plus important de comprendre les différents états de dégradation des biotopes (Menzies, 2000 ; Pooter et al., 2004 ; Tiokeng et al., 2015 ; Vroh et al., 2015). Cette compréhension passe par l'étude de la composition floristique et de la structure de la végétation (Odiwe et al., 2012). La structure de la végétation d'une forêt correspond à l'agencement de ses différents composants. Cet agencement peut être envisagé globalement ou séparément pour les différentes espèces (ou groupes d'espèces). Le mot structure a toujours suscité une polémique dans le monde pour la description des peuplements végétaux (Senterre, 2005). La structuration du peuplement arborescent constitue, en quelque sorte, l'ossature de la forêt.

En Côte d'Ivoire, la structure de la végétation des forêts n'a été abordée que sporadiquement (Kouamé et al., 2006). Concernant les forêts marécageuses, cette étude de la structure de la végétation de la Forêt Marécageuse de la Tanoé-Ehy (FMTE) est l'une des premières du genre. Cette forêt fait partir des zones dites humides qui subissent actuellement plusieurs menaces de la part de la population. Ceux sont entre autres, l'agriculture itinérante sur brûlis, la coupe d'arbres pour le charbon de bois et le bois de chauffage, la récolte des plantes médicinales ainsi que la coupe de bois pour la menuiserie (Zadou et al., 2011). Face à cette situation, des mesures pour la conservation de la FMTE s'avèrent indispensables pour sa survie car sa disparition, occasionnerait des pertes inestimables pour les populations riveraines et surtout pour le maintien de la biodiversité et du microclimat de la région. Les travaux de Missa et al. (2015) ont montré que la forêt possède certaines espèces végétales qui lui sont inféodées. L'objectif général est d'évaluer l'état de dégradation de la FMTE. De façon spécifique, il s'agit d'étudier les profils structuraux et les recouvrements des biotopes de la forêt des Marais Tanoé-Ehy.

\section{MATERIEL ET METHODES Site d'étude}

La Forêt marécageuse de la Tanoé-Ehy est située dans la région du Sud Comoé (Figure 1), précisément dans le département de Tiapoum, entre les latitudes $5^{\circ} 05^{\prime} 00^{\prime}$ ' et $5^{\circ} 15^{\prime} 00^{\prime}$ 'nord et les longitudes $2^{\circ} 45^{\prime} 00^{\prime}$ ' et $2^{\circ} 53^{\prime} 00^{\prime}$ 'ouest (Adou Yao, 2007). Cette forêt s'étend sur une superficie de 12000 ha. Elle est limitée à l'Ouest par la lagune Ehy, à l'Est et au Sud par le fleuve Tanoé. La FMTE est soumise à un climat de type subéquatorial à quatre saisons. Elle est très arrosée, en général, avec une pluviométrie moyenne annuelle comprise entre $1400 \mathrm{~mm}$ et 1600 $\mathrm{mm}$ (Brou et al., 2005). La température varie entre $22{ }^{\circ} \mathrm{C}$ et $30^{\circ} \mathrm{C}$ avec une moyenne de 26 ${ }^{\circ} \mathrm{C}$. La FMTE est située dans le secteur littoral, caractérisé par la présence des forêts marécageuses et des mangroves (Guillaumet et Adjanohoun, 1971). 


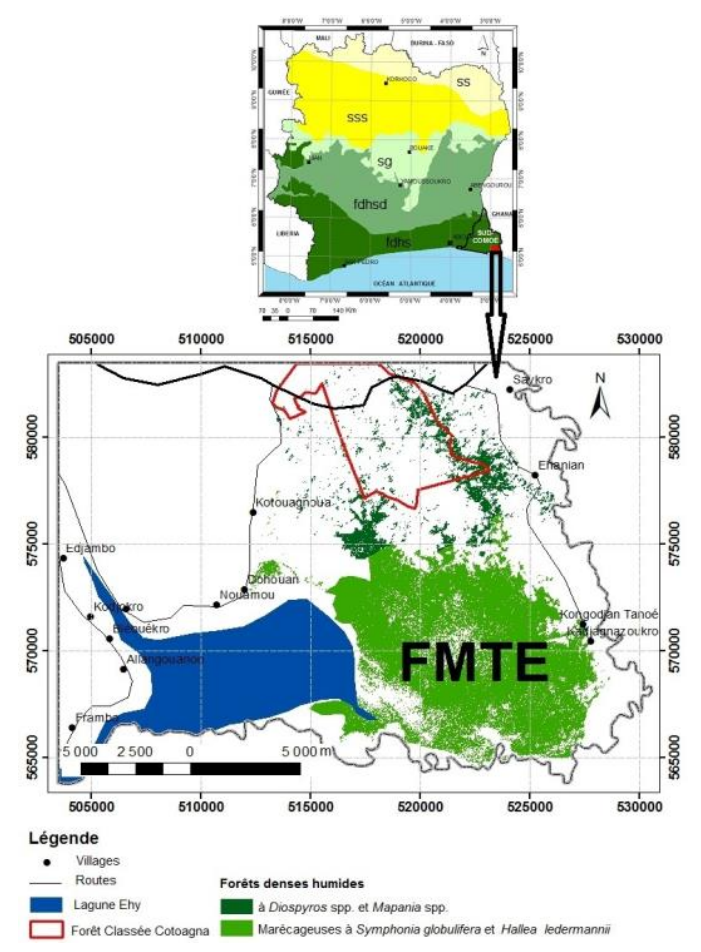

Figure 1: Carte de la végétation de la Côte d'Ivoire montrant la localisation de la forêt des Marais Tanoé-Ehy.

La FMTE est couverte en grande partie d'une végétation de forêt sempervirente, marécageuse sur un sol hydromorphe. Les autres formations végétales observées sont la raphiale et la forêt de terre ferme. Son relief est caractérisé par une frange littorale très peu élevée. Les altitudes sont au maximum de 10 à $12 \mathrm{~m}$ (Avenard et al., 1971). Dans la partie Sud de la FMTE, les sols sont organiques (argileux et limoneux). Selon Aubert (1965), de tels sols sont qualifiés de sols humides à Gley, salés ou sols de " poto-poto ». Ils se localisent dans des zones à écoulement difficile et sont difficilement praticables. En saison sèche, l'on a du mal à y circuler sans s'enfoncer dans la vase. Dans sa partie nord, les sols sont de plus en plus sablo-argileux, voire argileux à certains endroits (Bene et Akpatou, 2007).

\section{Méthode de terrain}

La méthode de relevé linéaire, proposée par Gautier et al. (1994), et déjà utilisée par plusieurs chercheurs en Côte d'Ivoire (Kouamé, 1998 ; Bakayoko, 1999) et à Madagascar (Messmer et Rakotomalaza, 1999; Messmer et al., 2000), a servi dans cette étude. Cette méthode consiste à tendre horizontalement à ras du sol une corde longue généralement 200 mètres (Chatelain, 1996 ; Bakayoko et al., 2011). À cause de la nature et de l'humidité des sols observés dans les zones marécageuses où l'on a du mal à circuler sans s'enfoncer et de la petite taille des forêts de terre ferme, nous avons utilisé une longueur de 100 mètres. À partir de ce dispositif, nous avons effectué 100 mesures à des intervalles réguliers d'un mètre. Ainsi, la hauteur minimale et maximale de chacun des contacts avec la végétation est mesurée à l'aide d'un jalon de $8 \mathrm{~m}$ dressé verticalement. Au-delà des $8 \mathrm{~m}$, les valeurs des hauteurs sont estimées. Dans la présente étude, au total 20 relevés linéaires ont été effectués dans la FMTE.

\section{Analyse des données}

Dans les analyses structurales, les profils structuraux et le recouvrement de la végétation ont été couramment utilisés 
(Bongers, 1988 ; Chatelain, 1996 ; Kouamé, 1998). Pour l'approche des profils structuraux, les structures horizontales ont été abordés. Elle se définit comme la représentation graphique de chaque relevé linéaire, sous la forme de profil structural, dans un plan défini par la longueur de la ligne du relevé, comme axe des abscisses, et la hauteur de la végétation, comme axe des ordonnées. Ces profils structuraux ont permis de comparer, visuellement, la structure de la végétation des biotopes de la forêt.

Afin de voir les différences de la structure verticale de la végétation des biotopes, à partir des données du relevé linéaire, les pourcentages de recouvrement dans les 6 intervalles de hauteur ont été définis par Emberger et al. (1968), soit:-<2 $\mathrm{m} ; 2$ à 4 $\mathrm{m} ; 4$ à $8 \mathrm{~m} ; 8$ à $16 \mathrm{~m} ; 16$ à $32 \mathrm{~m} ;>32$.

\section{RESULTATS}

\section{Structure horizontale}

Les données obtenues à partir des différents relevés effectués ont permi de caractériser la structure de la végétation de la forêt marécageuse. Concernant les profils structuraux (Figure 2), dans le relevé 1, un peuplement de Hallea ledermannii (K. Krause) Verdc. (Rubiaceae) dans les 15 premiers mètres et entre les $40^{\text {èmes }}$ et les $52^{\text {èmes }}$ mètres du relevé est observé ; cette espèce émerge au-dessus de la canopée. Ce relevé est très ouvert avec par endroit, une végétation qui dépasse à peine cinq mètres de hauteur. Dans le sous-bois de ce relevé, on observe Syzygium rowlandii Sprague (Myrtaceae) et Uapaca paludosa Aubrév. \& Leandri (Phyllanthaceae). Si la première espèce n'est rencontrée qu'entre les $20^{\text {èmes }}$ et $40^{\text {èmes }}$ mètres du relevé, la seconde est distribuée le long de tout le relevé. A partir des $50^{\text {ème }}$ mètres jusqu'aux $80^{\text {èmes }}$ mètres du relevé, nous notons une absence totale de végétation au niveau des strates moyennes et hautes. Le reste du profil est caractérisé par une canopée allant jusqu'à 30 mètres de hauteur avec une faible couverture de la végétation au niveau de la strate moyenne. L'observation des relevés R2 et R3 indique une faible hauteur de la végétation qui dépasse rarement les 25 mètres dans le relevé 2. Le sous-bois est pauvre dans la strate inférieure à deux mètres de hauteur. Ce relevé est caractérisé par Spondianthus preussii Engl. (Phyllanthaceae), Hallea ledermannii et la présence de liane au niveau du sous-bois. Sur le deuxième relevé de ce milieu, l'espèce Hallea ledermannii est rencontrée le long de tout le relevé. La végétation est homogène sur ce relevé avec très peu de trouées constatées. Comme au niveau du premier relevé, une absence de végétation dans les strates basses est remarquable. Cela est encore observable jusqu'à la strate moyenne. Les lianes qui étaient présentes en dessous de 10 mètres de hauteur sont moins nombreuses ici dans cette strate. Elles vont au-delà de 15 mètres au niveau du $55^{\text {ème }}$ et entre le $80^{\text {ème }}$ et le $90^{\text {ème }}$ mètre. Parmi les lianes, l'espèce Combretum racemosum P. Beauv. (Combretaceae) représente l'essentielle des espèces lianescentes sur le long du relevé. Le relevé 4 qui constitue le troisième relevé de ce milieu, est dominé par quatre espèces: Anthostema aubryanum Baill. (Euphorbiaceae), Uapaca guineensis, Raphia hookeri G. Mann \& H. Wendl. (Arecaceae) et Spondianthus preussii. Ce relevé est caractérisé par une pauvreté de la végétation au niveau de la strate moyenne. Le sous-bois n'est pas aussi fermé. Uapaca guineensis Mull. Arg. (Phyllanthaceae) et Raphia hookeri se retrouvent plus dans le sous-bois et ne vont qu'au-delà de 10 mètres de hauteur par endroits. Quant aux espèces Anthostema aubryanum et Spondianthus preussii, elles font partir des émergentes. Anthostema aubryanum émerge au-dessus de la canopée dès les premiers mètres du relevé et entre les $12^{\text {èmes }}$ et $25^{\text {èmes }}$ mètres du relevé où elle monte au-delà de 30 mètres de hauteur. L'espèce Spondianthus preussii domine également la canopée entre le $50^{\text {ème }}$ et le $57^{\text {ème }}$ mètre.

\section{Recouvrements}

L'analyse des recouvrements (Figure 3) indique que sur les quatre relevés, la végétation est dense dans les strates moyennes (strate de 8 à $16 \mathrm{~m}$ et de 16 à $32 \mathrm{~m}$ ) sauf pour les relevés R3. Les relevés 2 et 3 ont dans leur strate basse un taux de recouvrement en dessous de $40 \%$. Le taux de recouvrement croît progressivement comme un escalier renversé, jusqu'à la strate 8 à $16 \mathrm{~m}$ pour les relevés 2,3 et 4 . 

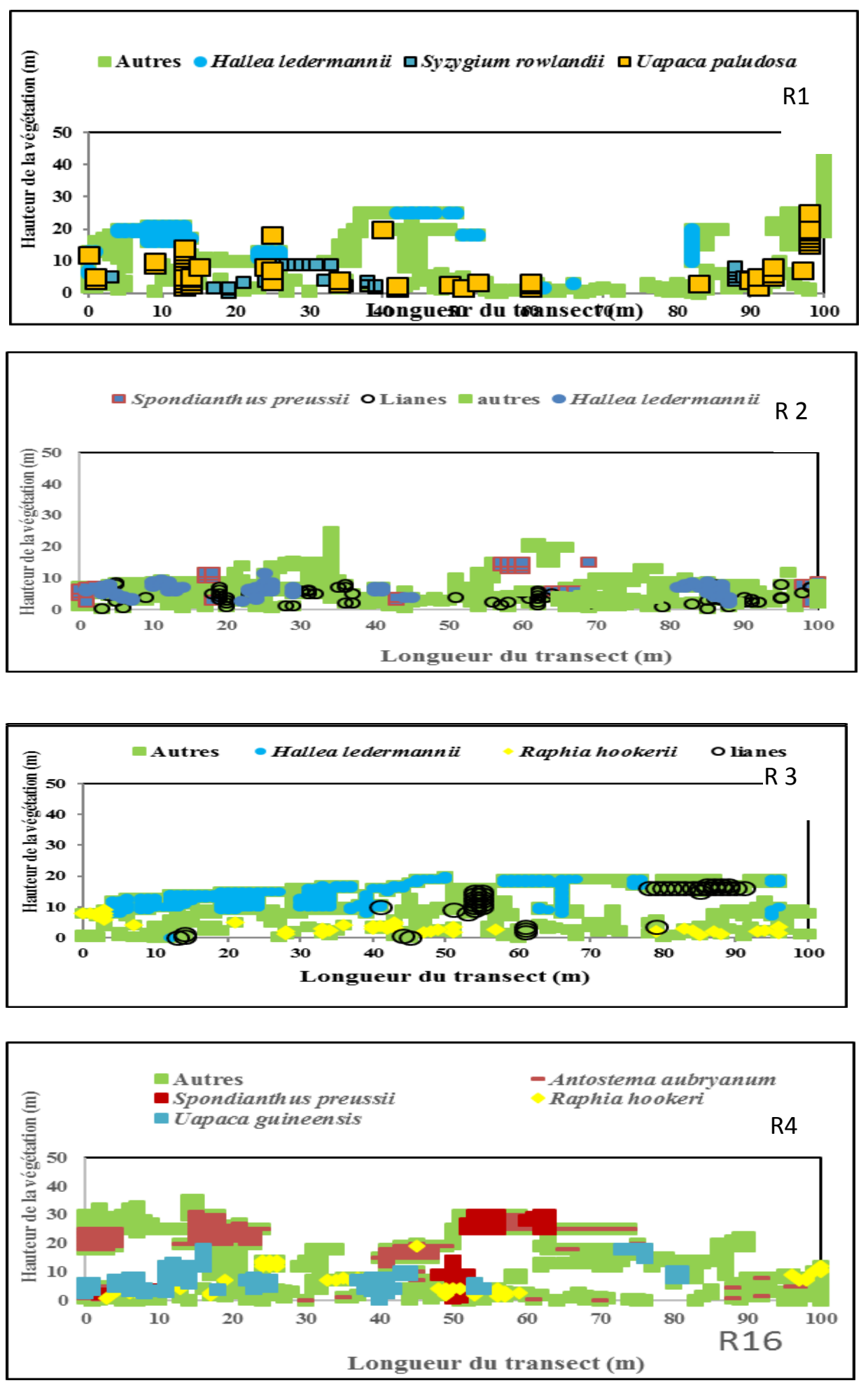

Figure 2 : Profils structuraux des relevés linéaires des zones marécageuses. 

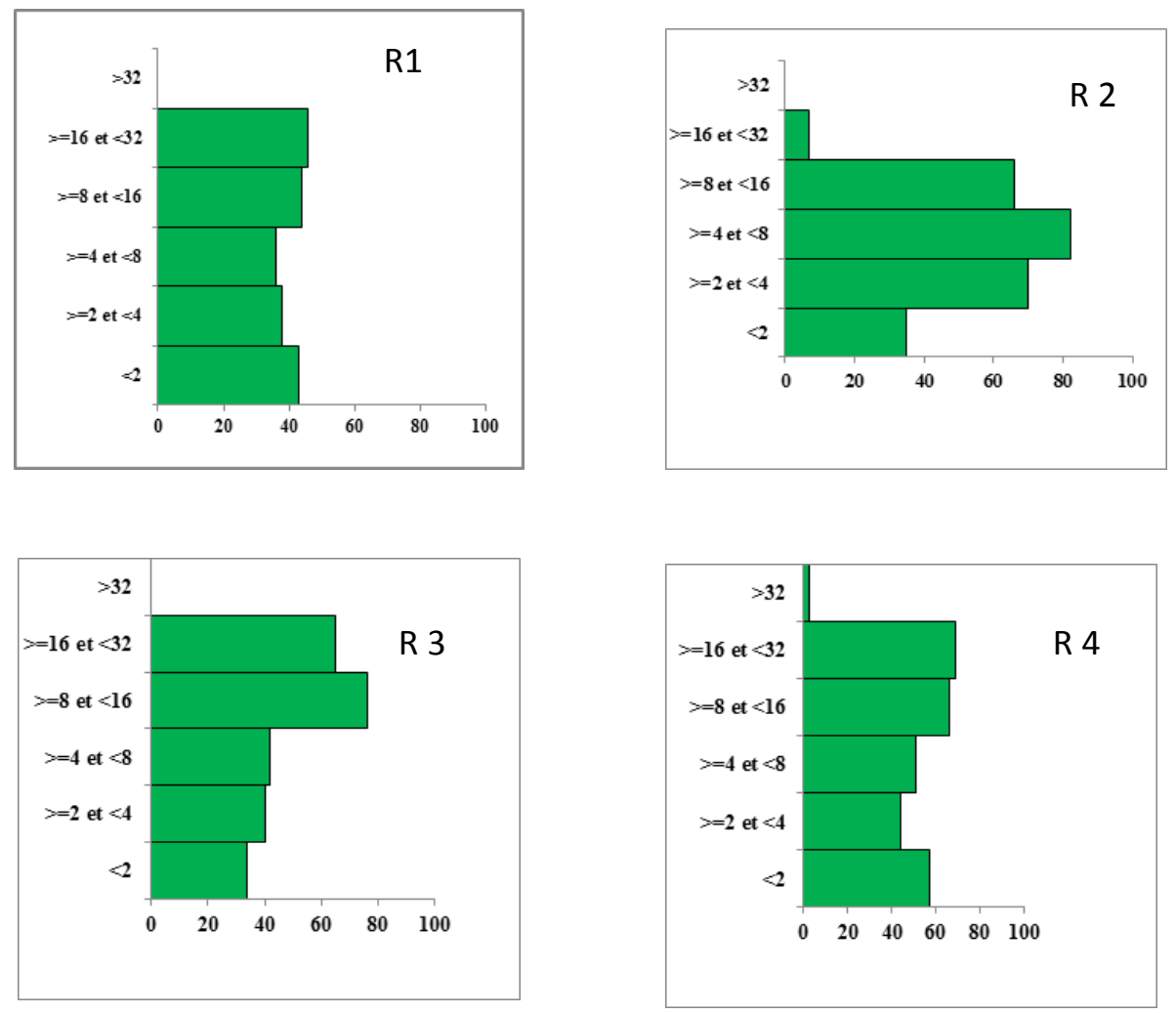

Figure 3 : Recouvrements des strates des relevés linéaires des zones marécageuses.

\section{DISCUSSION}

\section{Structure de la végétation}

L'analyse des profils structuraux indique que parmi les espèces qui contribuent fortement à la formation de la végétation des biotopes, nous avons la présence Hallea ledermanii qui est une espèce vulnérable selon l'IUCN (2018). Cette espèce est exploitée principalement par les populations provenant du Ghana, pays qui est séparé de la FMTE par le fleuve Tanoé. Cette observation montre que si la flore et la végétation de ces biotopes ne sont pas protégées, elles finiront par disparaître (Missa et al., 2015 ; André et al., 2016). Concernant la distribution des espèces, nous avons remarqué que l'espèce Anthostema aubryanum est moins présente. Cependant, certaines espèces se rencontrent dans tous les relevés. Des observations similaires ont été faites par Collinet (1997) en Guyane française. Il faut noter que ce n'est pas parce qu'une espèce se trouve de façon statistiquement significative dans une situation donnée, qu'elle préfère cette situation. En effet, le confinement de l'espèce dans les bas-fonds serait probablement dû à la compétition avec une ou plusieurs autres espèces qui réussissent mieux sur les sols bien drainés et repoussent vers les bas-fonds où l'espèce s'adapte mieux. Lorsque cette espèce concurrente n'est pas présente dans la localité, l'espèce occuperait les sols bien drainés (Pascale, 2003). Les biotopes présentent une proportion d'espèces moins importante au niveau de la strate inférieure. Ceci s'explique par la permanence d'eau sur le sol de la forêt marécageuse qui joue un rôle sélectif et qui rend de ce fait, le milieu lui-même pauvre en espèces. Cependant, plusieurs études montrent que les strates inférieures des milieux humides 
ont bien une plus grande diversité d'espèces grâce à la microtopographie qui prévaut au niveau du sol et qui permet à certaines d'entre elles de s'établir au-dessus de la nappe phréatique à certains endroits (Dabel et Day, 1977 ; Rheinhardt, 1992 ; Mata et al., 2011).

\section{Etat de la végétation}

Le recouvrement du relevé R2 est semblable au type G de Châtelain (1996) caractérisant les milieux très dégradés. Cette dégradation de la structure des milieux pourrait être due à l'exploitation villageoise qui a un grand impact sur la structure forestière (Bakayoko et al., 2011; Djègo et al., 2012). Le relevé R1, R2 et R4 présentent une structure particulière jamais observée en forêt dense humide de Côte d'Ivoire. Cela s'explique par l'aspect hydromorphe des sols. Selon Perraud (1971), le sol des forêts sempervirentes du Sud-Est appartient à la sous-classe des sols ferralitiques fortement désaturés du type granitique ou schisteux, ces sols ont une plus forte capacité de rétention en eau (De Rham, 1971).

\section{Conclusion}

Cette étude de la structure a fourni un niveau de connaissance sur l'état de conservation de la structure de la végétation de cette forêt. L'étude a permis de montrer que l'intégrité de cette forêt est menacée par les populations. Dans cette forêt, il existe une compétition entre les espèces des milieux humides. Ce biotope présente une proportion d'espèces moins importante au niveau de la strate inférieure. Dans ce biotope des structures particulières qui n'ont jamais été observées en forêt dense de Côte d'Ivoire y sont présentes. La présence de ces nouvelles structures est liée à l'hydromorphie des sols qui elle-même est traduite par des conditions environnementales particulières.

\section{CONFLIT D'INTERETS}

Les auteurs déclarent qu'il n'y a aucun conflit d'intérêts pour cet article.

\section{CONTRIBUTIONS DES AUTEURS}

MK s'est occupé de l'échantillonnage, traitement des données et de la rédaction du manuscrit. SF: échantillonnage, directive technique, correction du manuscrit et contribution financière. SD: directive technique, correction du manuscrit et contribution financière. BA: directeur scientifique de l'étude.

\section{REMERCIEMENTS}

Ce travail a bénéficié des financements du Programme d'Appui Stratégique à la Recherche Scientifique (PASRES) et du Centre Suisse de Recherches Scientifiques à travers le Programme Recherche et Action pour la Sauvegarde des Primates de Côte d'Ivoire (RASAP-CI). Nos remerciements vont également aux guides de terrain de la région pour avoir accepté de nous accompagner sur le terrain.

\section{REFERENCES}

Adou-Yao CY, N'Da DH, Bakayoko A, Kouassi KE, N'Guessan KE, Ake-Assi L. 2011. Distribution, régénération and conservation status of Nuxia congesta R. Br. ex Fresen. (Buddlejaceae) in Côte d'Ivoire. Am. $J$. Sci. Ind. Res., 2(5): 774-785. www.erudit.org/vertigo

Adou-Yao CY. 2007. Inventaire préliminaire de la flore et description de la végétation de la Forêt des Marais Tanoé. Rapport pour RASAP-CI, Abidjan, Côte d'Ivoire, $29 \mathrm{p}$.

André AD, Ngor N, Alla M, Bienvenu S, Cheikh SF, Lamine D, Cheikh M. 2016. Composition floristique et dynamique du parc agroforestier à Cordyla pinnata (Lepr. ex A. Rich.) Milne-Redh. dans le Sud du Bassin Arachidier (Sénégal). Int. J. Biol. Chem. Sci., 10(4): 1805-1822. DOI:

http://dx.doi.org/10.4314/ijbcs.v10i4.29

Aubert G. 1965. La Classification des Sols. Cahiers ORSTOM., 3: 269- 288. 
Aubreville A. 1959. Flore forestière de la Côte d'Ivoire. Centre Techn. For. Trop., 1-3: 310-186.

Avenard JM, Eldin M, Girad G, Sircoulon J, Touchebeuf P, Guillaumet JL, Adjanohoum E. 1971. Le Milieu Naturel de la Cote d'Ivoire. ORSTOM : Paris France.

Bakayoko A. 1999. Comparaison de la composition floristique et de la structure forestière des parcelles de la forêt classée de Bossématié, dans l'Est de la Côte d'Ivoire. Mémoire de DEA Ecol. Trop., Univ. De Cocody-Abidjan, Côte d'Ivoire, 72p.

Bakayoko A, Chatelain C, Martin P, Traoré D, Gautier L. 2011. Floristic study of some fragment forests in South Western of Cote d'Ivoire. European Journal of Scientific Research, 63(4): 468-481. http://www.eurojournals.com/ejsr.htm

Bakayoko A, Martin P, Gautier L, Chatelain C, Traoré D, Spichiger R. 2004. Etude comparative des massifs forestiers entourant la zone de Taï à Zagné (sudouest de la Côte d'Ivoire). Candollea, 59(2): 191-229.

Bene J-CK, Akpatou KB. 2007. Inventaire préliminaire de la faune avec un accent particulier sur les primates de la Forêt Journal of Animal \&Plant Sciences, 25(3): 3917-3938. http://www.m.elewa.org/JAPS

Brou YT, Akindès F, Bigot S. 2005. La variabilité climatique en Côte d'Ivoire : entre perceptions sociales et réponses agricoles. Cahiers Agricultures, 14: 533540.

Bongers F, Popma J, Del-Castillo JM, Carabias J. 1988. Structure and floristic composition of the lowland rain forest of Los Tuxtlas, Mexico. Végétation, 74: 5580.

Chatelain C. 1996. Possibilités d'application de l'imagerie satellitaire à haute résolution pour l'étude des transformations de la végétation en Côte d'Ivoire forestière. Thèse Doct. Ès Sc. Nat. Fac. Sc., Univ. Genève, 206 p.

Collinet F. 1997. Essai de regroupement des principales espèces structurantes d'une forêt dense humide d'après l'analyse de leur répartition spatiale (forêt de Paracou Guyane). Thèse de doctorat, Université Claude Bernard, Lyon1, 313 p.

Dabel CV, Day FP. 1977. Structural comparisons of four plant communities in the Great Dismal Swamp, Virginia. Bulletin of the Torrey Botanical Club, 104(4): 352-336.

Devineau JL. 1973. Etude quantitative des forêts galeries de Lamto (généralités. Bull. Liaison Chercheurs Lamto, 19-22.

De Rham P. 1971. L'azote dans quelques forêts, savanes et terrains de culture d'Afrique tropicale humide (Côte d'Ivoire). Thèse Doctorat ès Sciences, Université de Lausanne, Lausanne, 124 p.

Djègo J, Gibigaye $\mathrm{M}$, Tente $\mathrm{B}$, Sinsin $\mathrm{B}$. 2012. Analyse écologique et structurale de la forêt communautaire de Kaodji au Bénin. Int. J. Biol. Chem. Sci., 6(2): 705713. DOI: http://dx.doi.org/10.4314/ijbcs.v6i2.14

Emberger L, Godron M, Daget P. 1968. Code Pour le Relevé Méthodique de la Végétation et du Milieu; Principes et Transcription sur Cartes Perforees. Editions CNRS: Paris.

Rheinhardt RD. 1992. A multivariate-analysis of vegetation patterns in tidal fresh-water swamps of lower Chesapeake Bay, USA. Bulletin of the Torrey Botanical Club, 119(2): 192-207.

Gautier L, Chatelain C, Spichiger R. 1994. Presentation of a releve for vegetation studies based on high resolution satellite imagery: comptes rendus de la treizième réunion plénière de l'AETFAT, Zomba, 
Malawi. Nat. Herb. Bot. Gard. Malawi. 2: 1339-1350.

Gueulou N, Ouattara ND, Konan D, Gnahoré E, Missa K, Bakayoko A. 2018. Diversité floristique et structurale de la forêt galerie du Bandama dans la Réserve Scientifique de Lamto en Côte d'Ivoire. Afrique Science, 14(4): 452439. http://www.afriquescience.ney

Guillaumet J-L, Adjanohoun E. 1971. La végétation de la Côte d'Ivoire. In Le Milieu Naturel de la Côte d'Ivoire, Avenard JM, Eldin E, Girard G, Sircoulon J, Touchebeuf P, Guillaumet JL, Adjanohoun E, Perraud A (eds). ORSTOM : Paris ; 157- 266.

Kouamé D, Adou Yao CY, Kouassi KE, N'Guessan KE. 2008. Preliminary floristic Inventory and Diversity in Azagny National Park (Côte d'Ivoire). European journal of Scientific Research, 23(4): 537-547.

http://www.eurojournals.com/ejsr.htm

Lebrun J-P, Stork AL. 1991. Enumération des plantes à fleurs d'Afrique Tropical: Généralités et Anonaceae à Pandaceae (vol I). Edition des Conservatoires et jardin botaniques : Genève-Suisse.

Koné I, Béné J-CK, N'guessan AK, Bitty AE, Koffi AD, Akpatou KB, Gonedelébi S. 2008. Plaidoyer pour la sauvegarde de la Forêt des Marais Tanoé (Sud-est de la Côte d'Ivoire), un site à valeur de conservation exceptionnelle en Afrique de l'Ouest et dans le monde. RASAPCI, Abidjan, Côte d'Ivoire, 25p.

Kouamé FNG. 1998. Influence de l'exploitation de l'exploitation forestière sur la végétation et la flore de la Forêt Classée du Haut Sassandra (Centre Ouest de la Côte d'Ivoire). Thèse de $3^{\text {eme }}$ cycle, Université de Cocody, Abidjan, $227 \mathrm{p}$.

Kouamé FNG, Bakayoko A, Traoré D. 2006. Caractéristiques structurales de la forêt dense humide de basses altitudes de la côte d'ivoire. Rev. Ivoir. Sci. Technol., 08: $17-195$

Mata DIP, Moreno-Casasola C, Madero-Vega G, Castillo-Campos, Warner BG. 2011. Floristic composition and soil characteristics of tropical freshwater forested wetlands of Veracruz on the coastal plain of the Gulf of Mexico. Forest Ecology and Management. 262(8): 1514-1531. DOI //link.springer.com/

Missa K, Ouattara ND, Koné M, Bakayoko A. 2015. Étude floristique et diversité de la forêt des MaraisTanoé-Ehy (Sud- Est de la Côte d'Ivoire: 2015. Journal of Animal \&Plant Sciences, 25(3): 39173938. http://www.m.elewa.org/JAPS

Messmer N, Rakotomalaza P-J. 1999. Étude de la structure et de la composition floristique de la végétation. In Inventaire Biologique de la Réserve Spéciale du Pic d'Ivohibe et du Couloir Forestier qui la Relie au Parc National d'Andringitra, Goodman S, Rasolonandrasana BPN (eds). CIDST: Antananarivo; 43-80.

Messmer N, Rakotomalaza P-J, Gautier L. 2000. Structure and floristic composition of the vegetation of the Parc National de Marojejy, Madagascar. In A floral and faunal inventory of the Parc National de Marojejy, Madagascar: With Reference to Elevational Variation, Messmer N, Rakotomalaza P-J, Gautier L (ed). Fieldiana-Zoology: New Ser.; 41-104.

Menzies A. 2000. Structure et composition floristique de la forêt de la zone Ouest du Parc nationale de Tai (Côte d'Ivoire). Mémoire, Université de Genève, 124 p.

Odiwe AI, Adewumi RA, Alimi AA, Olugbenga OO. 2012. Carbon stock in topsoil, standing floor litter and above ground biomass in Tectona grandis plantation 10-years after establishment in Ile-Ife, Southwestern Nigeria. Int. J. 
Biol. Chem. Sci., 6(6): 3006-3016. DOI: http://dx.doi.org/10.4314/ijbcs.v6i6.3

Pascal J-P. 2003. Description et dynamique des milieux forestiers. Rev. For. Fr. $L V$, 118- 130.

Perraud. 1971. Les sols de la Côte d'Ivoire. In Le Milieu Naturel de la Côte d'Ivoire, Perraud (ed). ORSTOM: Paris (France); 269-389.

Pooter L, Bongers F, Kouamé F, Hawthorne WD. 2004. Biodiversity of West African Forest: An Ecological Atlas of Woody Plant Species. CABI Publishing: London-UK.

Tiokeng B, Mapongmetsem PM, Nguetsop VF, Tacham WN. 2015. Biodiversité floristique et régénération naturelle sur les Hautes Terre de Lebialem (Ouest Cameroun). Int. J. Biol. Chem. Sci., 9(1): 56-68.

DOI:

http://dx.doi.org/10.4314/ijbcs.v9i1.6
Vroh Bi TAA, Kouame Y, N'da D, N'guessan K. 2010. Diversité floristique et structurale sur le Site d'une Réserve Naturelle Volontaire à Azaguié, Sud-est de la Côte d'Ivoire. European Journal of Scientific Research, 45(3): 411-421. http://www. Euro. journals. com/ejsr.htm

IUCN. 2018. Red List of Threatened Species. IUCN. http://www.iucnredlist. org/. Consulted on March 2018.

Zadou DA, Koné I, Mouroufié VK, Adou Yao CY, Gleanou EK, Kablan YA, Coulibaly D, Ibo JG. 2011. Valeur de la forêt des Marais Tanoé-Ehy (Sud-Est de la Côte d'Ivoire) pour la conservation: dimension socio-anthropologique. Tropical Conservation Science, 4(4): 373-385. DOI:

https://doi.org/10.1177\%2F19400829110 0400402 